CONFORMAL GEOMETRY AND DYNAMICS

An Electronic Journal of the American Mathematical Society

Volume 5, Pages 1-5 (February 21, 2001)

S $1088-4173(01) 00066-2$

\title{
DIFFEOMORPHISMS OF THE CIRCLE AND HYPERBOLIC CURVATURE
}

\author{
DAVID A. SINGER
}

\begin{abstract}
The trace $T f$ of a smooth function $f$ of a real or complex variable is defined and shown to be invariant under conjugation by Möbius transformations. We associate with a convex curve of class $C^{2}$ in the unit disk with the Poincaré metric a diffeomorphism of the circle and show that the trace of the diffeomorphism is twice the reciprocal of the geodesic curvature of the curve. Then applying a theorem of Ghys on Schwarzian derivatives we give a new proof of the four-vertex theorem for closed convex curves in the hyperbolic plane.
\end{abstract}

\section{INTRODUCTION}

In a recent note ([2]), Duval and Ovsienko, together with L. Guieu, related the Schwarzian derivative to the geometry of Lorentz surfaces. They observed that for a curve $y=f(x)$ in the plane with the metric $g=d x d y$, the Lorentz curvature is given by the quantity

$$
\varrho(x)=\frac{f^{\prime \prime}(x)}{f^{\prime}(x)^{3 / 2}} .
$$

The curvature satisfies

$$
\sqrt{f^{\prime}(x)} \varrho^{\prime}(x)=S f(x)
$$

where

$$
S f(x)=\frac{f^{\prime \prime \prime}(x)}{f^{\prime}(x)}-\frac{3}{2} \frac{f^{\prime \prime}(x)^{2}}{f^{\prime}(x)^{2}}
$$

is the Schwarzian derivative of $f$. They then apply a theorem of Ghys ([3], [6]): If $f$ is a diffeomorphism of $\mathbb{R} P^{1}$, then the Schwarzian derivative $S f$ has at least four distinct zeroes. This implies the four-vertex theorem for timelike closed curves in constant curvature Lorentz surfaces.

In this note we derive analogous results for the (Riemannian) hyperbolic plane, using a function related to $\varrho(x)$. We then apply the theorem of Ghys to give a proof of the four-vertex theorem for closed convex curves in the hyperbolic plane.

Received by the editors July 26, 2000 and, in revised form, January 23, 2001.

2000 Mathematics Subject Classification. Primary 53A55; Secondary 52A55.

(C)2001 American Mathematical Society 


\section{The HYPERBolic TRACE}

If $w=f(z)$ is a differentiable function, of class at least $C^{2}$, of one real or complex variable, define its hyperbolic trace $T f$ by

$$
T f(z)=\frac{1}{2} \frac{f^{\prime \prime}(z)}{f^{\prime}(z)^{3 / 2}}(z-f(z))+\frac{\left(f^{\prime}(z)+1\right)}{\sqrt{f^{\prime}(z)}} .
$$

Then

$$
(T f)^{\prime}(z)=\frac{z-f(z)}{2}\left(\frac{f^{\prime \prime \prime}(z)}{f^{\prime}(z)^{3 / 2}}-\frac{3}{2} \frac{f^{\prime \prime}(z)^{2}}{f^{\prime}(z)^{5 / 2}}\right)=\frac{z-f(z)}{2 f^{\prime}(z)^{1 / 2}} S f(z) .
$$

From this it follows immediately that $T f(z)$ is constant when $f$ is a Möbius transformation. More precisely,

Lemma 2.1. If $f(z)=\frac{a z+b}{c z+d}$, with $a d-b c=1$, then $T f(z)=a+d$.

If we represent the group of Möbius transformations as $P S L(2)$, then $f$ corresponds to the matrix

$$
\left(\begin{array}{ll}
a & b \\
c & d
\end{array}\right)
$$

and $T f$ is just the trace. More precisely, $T f$ is the ratio of the trace to the square root of the determinant, which is a conjugacy invariant on $P S L(2)$. This fact is actually a special case of a much more general result:

Theorem 2.2. The hyperbolic trace is a conjugacy invariant under Möbius transformations. If $R$ is a Möbius transformation, if $g$ is defined by $g(R(z))=R(f(z))$, and if $w=R(z)$, then $T g(w)=T f(z)$.

This can be verified by a straightforward computation:

Case 1: $w=a z+b$; verification is simple.

Case 2: $w=\frac{1}{z}$; one can verify that

$$
\frac{1}{2} \frac{g^{\prime \prime}(w)}{g^{\prime}(w)^{3 / 2}}(w-g(w))=\frac{1}{2} \frac{f^{\prime \prime}(z)}{f^{\prime}(z)^{3 / 2}}(z-f(z))+\frac{z-f(z)}{z \sqrt{f^{\prime}(z)}}-\frac{(z-f(z)) \sqrt{f^{\prime}(z)}}{f(z)}
$$

from which the result quickly follows.

Now the general case may be deduced from these cases by composition.

Remark 2.3. The hyperbolic trace is uniquely characterized by Lemma 2.1 among second-order differential operators. There is an ambiguity in the definition of $T f$ caused by the necessity to choose a branch of the square root. Correspondingly, there is an ambiguity in the choice of $\frac{\operatorname{tr} A}{\sqrt{\operatorname{det} A}}$ for $A$ in $P S L(2, \mathbb{C})$.

The following is an easy exercise:

Proposition 2.4. If $w=f(z)$ has inverse $z=g(w)$, then $T f(z)=T g(w)$.

\section{Hyperbolic CONVEX CURVES}

In this section we consider the Poincaré disk model of the hyperbolic plane $\mathbb{H}$. Let $S^{1}=\{z \in \mathbb{C}|| z \mid=1\}$ be the unit circle in $\mathbb{C}$, thought of as the set of ideal points of $\mathbb{H}$. We consider diffeomorphisms of $S^{1}$ and their traces calculated with respect to conformal coordinate systems on $S^{1}$, i.e., arising from the three-parameter family of Möbius transformations from $S^{1}$ to circles or lines in the Riemann sphere. In particular, the usual parametrization by $\theta \bmod 2 \pi$ is not an allowed coordinate 
system. The point is that using $\theta$ corresponds to conjugating $f$ by an exponential function. For example, the rotation $z \longrightarrow e^{2 i \pi \alpha} z$ is given by multiplication by a constant; the corresponding transformation $\theta \longrightarrow \theta+\alpha$ is given by addition of a constant. These two functions have different hyperbolic traces.

Let $\gamma(t)$ be a smooth regular curve in $\mathbb{H}$. For each $t$, the curve determines a unique pair of ideal points $(\alpha(t), \omega(t))$ in $S^{1}$ such that the geodesic in $\mathbb{H}$ from $\alpha(t)$ to $\omega(t)$ is the oriented tangent line to $\gamma$ at $t$. Thus a regular curve $\gamma$ in $\mathbb{H}$ determines a curve $\bar{\gamma}$ in the space $\Sigma=S^{1} \times S^{1}-\triangle$, where $\triangle$ is the diagonal.

Although we will not use the following observation in this note, it is useful to consider $\Sigma$ as a Lorentzian manifold with the natural metric $g=d x d y$. In that case, following [2], if the resulting curve is given as the graph of $y=f(x)$, then formula (1.1) says that if $f^{\prime}(x) \neq 0$, then $\varrho(x)$ is the curvature of the curve. The construction of $\bar{\gamma}$ naturally generalizes to higher dimensions. Thus, for example, if $M$ is an oriented surface in hyperbolic three-space, then its tangent plane at each point determines an oriented ideal circle in the unit sphere. The space of oriented circles in the sphere is naturally isomorphic to the homogeneous space $P S L(2, \mathbb{C}) / P S L(2, \mathbb{R})$, which has a natural equivariant Lorentzian metric. Thus a surface $M$ gives rise to a surface $\bar{M}$ in $\operatorname{PSL}(2, \mathbb{C}) / P S L(2, \mathbb{R})$, with congruent surfaces corresponding to congruent surfaces.

Now in the case where $\gamma$ is a closed, convex curve in $\mathbb{H}$, the corresponding curve $\bar{\gamma}$ is the graph of a diffeomorphism $f$ of the unit circle. Note that an isometry of $\mathbb{H}$ is given by a Möbius transformation of the complex plane. This shows that isometric curves give rise to diffeomorphisms of the circle which are conjugate by elements of $P S L(2)$.

Example 3.1. Let $\epsilon_{r}$ denote the circle of hyperbolic radius $r$ centered at the origin. From elementary differential geometry, this circle has Euclidean radius

$$
R=\tanh \frac{r}{2}
$$

and geodesic curvature $K>1$ given by

$$
K=\operatorname{coth} r=\frac{1+R^{2}}{2 R} .
$$

A geodesic tangent to $\epsilon_{r}$ is a circle of Euclidean radius A centered at a point whose Euclidean distance from the origin is $A+R$. Since this circle is orthogonal to the unit circle, it is easy to see that $A=\frac{1-R^{2}}{2 R}$. From this it follows quickly that the corresponding diffeomorphism is the rotation $f(z)=e^{2 i \phi} z$, where $\tan (\phi)=A=$ $\frac{1-R^{2}}{2 R}$, but then

$$
T f=e^{i \phi}+e^{-i \phi}=2 \cos (\phi)=\frac{2}{A+R}=\frac{4 R}{1+R^{2}}=\frac{2}{K} .
$$

Thus, by Theorem 2.2 for any circle of curvature $K$ in $\mathbb{H}$, the corresponding diffeomorphism $f$ has $T(f) \equiv \frac{2}{K}$.

Example 3.2. Let $h$ be a horocycle, which is a curve of constant geodesic curvature 1. In the Poincaré disc, this curve is represented by a circle tangent to the unit circle. It is awkward to compute the map $f$ directly, but it becomes easy if we transform the problem to the upper half-plane. In this model, we may take for our horocycle a horizontal line $y=y_{0}>0$. The tangent lines to $h$ are semicircles centered on the $x$-axis; these obviously all have the same radius. Thus the diffeomorphism of 
the ideal circle, which in this model is the $x$ - axis, is a translation $\hat{f}(z)=z+c$, where $c= \pm y_{0}$. The matrix of $\hat{f}$ is $\left(\begin{array}{ll}1 & c \\ 0 & 1\end{array}\right)$, and conjugation with $\left(\begin{array}{ll}1 & i \\ i & 1\end{array}\right)$ gives the matrix for $f:\left(\begin{array}{cc}2+i c & c \\ c & 2-i c\end{array}\right)$. Again $T(f)=T(\hat{f}) \equiv 2=\frac{2}{K}$.

Example 3.3. Let $e$ be an equidistant curve, which is a curve of constant curvature $K, 0<K<1$. Again it is simpler to do the computation first in the upper half-plane. This time we may take the straight line $y=m x$, which has $K=\frac{1}{\sqrt{1+m^{2}}}$. The tangent line to the point $(x, m x)$ joins the points $\alpha(x)=$ $\left(\sqrt{m^{2}+1}-m\right) \sqrt{m^{2}+1} x$ and $\omega(x)=\left(\sqrt{m^{2}+1}+m\right) \sqrt{m^{2}+1} x$. The transformation $\hat{f}$ is therefore the dilation $\hat{f}(x)=\frac{\sqrt{m^{2}+1}+m}{\sqrt{m^{2}+1}-m} x$. The corresponding matrix is $\left(\begin{array}{cc}\sqrt{m^{2}+1}+m & 0 \\ 0 & \sqrt{m^{2}+1}-m\end{array}\right)$. Again we verify that $T(f)=2 \sqrt{m^{2}+1}=2 / K$.

Now if $\gamma(t)$ is any curve of class $C^{2}$ in $\mathbb{H}$, we can approximate it at a point $\gamma(t)$ to second order by a curve of constant curvature, which we will call the osculating circle. It follows that the value of $T f(\alpha(t))$ is the same as that of the osculating circle. We have proved

Theorem 3.4. For any simple closed curve $\gamma(t)$ in $\mathbb{H}$ with nonvanishing curvature $K(t)$, the corresponding diffeomorphism $f$ satisfies

$$
T f(\alpha(t))=\frac{2}{K(t)}
$$

Remark 3.5. Proposition 2.4 becomes, in this context, the statement that we may replace $f$ by its inverse in the theorem above.

\section{THE FOUR-VERTEX THEOREM}

The classical four-vertex theorem states that a simple closed curve in the plane of class $C^{2}$ with nonvanishing curvature has at least four extrema of the curvature. It was first published by Mukhopadhyaya in 1909 ([5]), and many generalizations have appeared over the years. The four-vertex theorem for convex curves in simplyconnected surfaces of constant curvature was proved by Jackson (4]). Another proof may be found in a paper of Cairns and Sharpe ([1]), and the theorem has been generalized to higher dimensional hyperbolic spaces by Uribe-Vargas ([8]). Scherk observed as early as 1945 ([7]) that the result that stereographic projection can be used to transfer problems about vertices of plane curves to problems about curves on the sphere, and Cairns and Sharpe observed that the property of being a vertex is a conformal invariant. The four-vertex theorem holds for simple closed, not necessarily convex, curves but is false for nonsimple curves, including locally convex curves.

Theorem (Ghys' Theorem ([2],[3])). If $f$ is a diffeomorphism of the real projective line $\mathbb{R} P^{1}$, then the Schwarzian derivative $S f$ has at least four distinct zeroes.

Combining Theorem 3.4 and formula (2.2), we see that

$$
K^{\prime}(t)=\frac{K(t)^{2} \alpha^{\prime}(t)(\omega(t)-\alpha(t))}{4 \sqrt{f^{\prime}(\alpha(t))}} S f(\alpha(t)) .
$$

This vanishes precisely when $S f(\alpha(t))$ vanishes. Therefore Ghys' theorem implies the four-vertex theorem in hyperbolic space. 


\section{REFERENCES}

1. G. Cairns and R.W. Sharpe, On the inversive differential geometry of plane curves, Enseign. Math. (2) 36 (1990), no. 1-2, 175-196. MR 91h:53001

2. C. Duval and V. Ovsienko, Lorentz world lines and Schwarzian derivative, (Russian) Funktsional. Anal. i Prilozhen. 34 (2000), no. 2, 69-72; translation in Funct. Anal. Appl. 34 (2000), no. 2, 135-137. CMP 2000:17

3. E. Ghys, Cercles osculateurs et géométrie Lorentzienne, Colloquium talk at Journée inaugurale du CMI, Marseille, February 1995.

4. S.B. Jackson, The four-vertex theorem for surfaces of constant curvature, Amer. J. Math. 67 (1945), 563-582. MR 7:259h

5. S. Mukhopadhyaya, New methods in the geometry of a plane arc, Bull. Calcutta Math. Soc. 1 (1909), 31-37.

6. V. Ovsienko and S. Tabachnikov, Sturm theory, Ghys theorem on zeroes of the Schwarzian derivative and flattening of Legendrian curves, Selecta Math. (N.S.) 2 (1996), no. 2, 297-307. MR 98g:57050

7. P. Scherk, The four-vertex theorem, Proceedings of the First Canadian Mathematical Conference (Montreal), 1945, Toronto, 1946, pp. 97-102. MR 8:485d

8. Ricardo Uribe-Vargas, On the $(2 k+2)$-vertex and $(2 k+2)$-flattening theorems in higherdimensional Lobatchevskian space, C. R. Acad. Sci. Paris Sér. I Math. 325 (1997), 505-510. MR 2000e:51027

Department of Mathematics, Case Western Reserve University, Cleveland, Ohio 44106-7058

E-mail address: das50po.cwru.edu 